\title{
LA VENGANZA DE HUME. UNA DEFENSA DEL EMOTIVISMO MORAL
}

CAMILO SEPÚLVEDA BETANCURTH

Universidad Autónoma de Manizales, Colombia

(c) $\underset{\mathrm{Br}}{\mathrm{gr}}$ 


\section{LA VENGANZA DE HUME. UNA DEFENSA DEL EMOTIVISMO MORAL*}

Resumen: Me propongo, en este trabajo, hacer una breve exposición de la teoría moral de Hume y sobre el emotivismo moral positivista. Presentaré algunas de las objeciones más fuertes a dichas teorías, las que por poco las dejan fuera de combate; y, por último, intentaré mostrar que los hallazgos que dan cuenta del funcionamiento de la naturaleza moral humana, desde un punto de vista neuronal, parecen ajustarse a los presupuestos más básicos de ambas teorías, sin que su compatibilidad sea total. Para ello me valdré de un ejemplo: el asesinato de un sujeto y la posterior declaración de los testigos.

Palabras claves: Hume, emotivismo moral, racionalismo moral, neuroética, positivismo.

\section{THE HUME'S REVENGE. A DEFENCE OF THE MORAL EMOTIVISM}

Abstract: My porpose, in this paper, is to give a brief exposition of the Hume's moral theory, and the positivist moral emotivism. I will present some of the strongest objections of both theories, which narrowly leave them out of combat. Finally, that the findings that give account of the functioning of the human moral nature, from a neuronal point of view, seem to fit the most basic presuppositions of both theories, without their compatibility being total. For this I will use an example: the murder of a subject and the subsequent statement of witnesses.

Keywords: Hume, Moral Emotivism, Moral Rationalism, Neuroethics, Positivism.

\section{Fecha de recepción: septiembre 20 de 2016 \\ Fecha de aceptación: febrero 6 de 2017}

Forma de citar (APA): Sepúlveda, C. (2017). La venganza de Hume. Una defensa del emotivismo moral. Revista Filosofía UIS. 16 (1), doi: http://dx.doi.org/10.18273/ revfil.v16n1-2017004

Forma de citar (Harvard): Sepúlveda, C. (2017). La venganza de Hume. Una defensa del emotivismo moral. Revista Filosofía UIS. 16 (1), 79-92.

Camilo Sepúlveda Betancurth: colombiano. Magíster en Filosofía Universidad de Caldas. Profesor Universidad Autónoma de Manizales.

Correo electrónico: camilosepul@gmail.com, camilosepulveda@autonoma.edu.co

\footnotetext{
"Artículo de revisión de tema.
} 


\section{LA VENGANzA de hUME. UNA defENSA DEL EMOTIVISMO MORAL}

Si por algo no es recordado Hume es por su filosofía moral. Difícilmente se hará alguna referencia a él en los compendios de ética y si se le menciona, es solamente para dejar registro de que Hume algo dijo acerca del tema.

A pesar de haber dedicado su tercer libro del tratado sobre la naturaleza humana (2011) a desarrollar una teoría moral y de contar en su haber con algunos ensayos en ese mismo sentido, sería muy generoso decir que el filósofo de Edimburgo dejó algún legado en la materia. Solamente un sector del positivismo lógico, representado sobre todo por Ayer y Stevenson, se propuso refinar la teoría humeana en lo que ellos Ilamaron emotivismo moral, que se derivaba de su teoría del significado del mismo positivismo; y salvo algunos trabajos de Von Wrigth en los años cincuenta, la teoría emotivista sufriría el mismo destino que la teoría que la inspiró: el casi absoluto ostracismo. De hecho, si por algo no se recuerda al positivismo lógico es por su filosofía moral.

Sin embargo, descubrimientos hechos en neurociencia y en sicología moral desde los años ochenta parecen indicar que la teoría moral humeana, y la posterior teoría emotivista del positivismo lógico, tienen fundamentos bastante sólidos y parecen dar una explicación bastante convincente sobre la naturaleza de la moral humana. Veamos el siguiente ejemplo:

\section{EL CRIMEN}

Imaginemos el siguiente escenario: dos hombres presencian cómo un asesino ultima a su víctima propinándole varios disparos en el pecho. Como sería de esperarse, los testigos del crimen, condenan, casi de manera automática, la acción. Las autoridades, una vez llegan al lugar de los hechos, entrevistan e interrogan a los dos testigos, para tener mayor información que los conduzca al esclarecimiento del homicidio. 
Así, el primer hombre declara que el asesino disparó tres veces sobre su víctima, con lo cual, su compañero está de acuerdo. Luego informa que, aparentemente, el asesinado perdió la vida de manera inmediata. Posteriormente sugiere que el arma homicida es un revolver, y no una pistola como había dicho inicialmente el otro testigo. Los dos testigos discuten acerca de detalles, y se dan cuenta que coinciden en casi todos, pero no en todos. En lo único en lo que ambos parecen estar completamente de acuerdo, es en que condenan el asesinato como reprobable moralmente; es decir, consideran que el asesinato es malo.

Un análisis detallado de los enunciados que profirieron los testigos arrojará, rápidamente, una clara diferencia entre los enunciados descriptivos y los enunciados prescriptivos. Así, los enunciados descriptivos hacen referencia al mundo, a los hechos del mundo, mientras que los enunciados prescriptivos hacen referencia a la valoración que hacemos sobre los hechos del mundo. La información objetiva que los testigos proporcionaban a las autoridades son, por supuesto, enunciados descriptivos, que pueden ser corroborados (así sea de manera hipotética), por los encargados de investigar el crimen. Por ejemplo, el enunciado, "el asesino disparó tres veces" describe un estado de cosas que puede ser verdadero o falso, y que puede ser corroborable empíricamente. Bastará, para su adecuación, en términos de verdad o falsedad, con que los peritos revisen el número de agujeros que tiene la víctima en su cuerpo, o el número de casquillos de bala esparcidos por la escena del crimen. Sin embargo, el enunciado "matar es malo", es claramente, de naturaleza diferente a los enunciados que simplemente describen los hechos en el mundo.

Si bien la teoría emotivista se inaugura propiamente como resultado de las hipótesis del positivismo lógico, particularmente con el trabajo de A.J. Ayer y Ch. L. Stevenson, en la propuesta filosófica humeana se puede encontrar algunos elementos que posteriormente serán heredados por el mismo positivismo.

Según Hume, solamente existen dos operaciones del entendimiento humano a través de las cuales podemos conocer algo: el conocimiento de los hechos del mundo, y el conocimiento de las relaciones de ideas. Por tanto, si pudiéramos, a través de la razón, conocer las distinciones morales, tendría que ser a través de uno de estos dos medios. Sin embargo, para la posición empirista de filósofo de Edimburgo, la posibilidad de que "lo bueno" o "lo malo", pueda ser aprehendido a través de nuestro contacto con el mundo es imposible, ya que, cuando presenciamos un hecho que condenamos o aprobamos moralmente, no presenciamos en él ninguna característica empírica que nos haga darle una valoración moral, es decir, entre las impresiones que recibimos de la acción, no se encuentran la bondad o la maldad. Para el caso del ejemplo del cual nos estamos valiendo, los testigos pueden, a través de la percepción, recibir las impresiones empíricas del número de disparos, de las condiciones climáticas bajo las cuales se cometió el crimen, del tipo de arma que se usó, de las características físicas de 
la víctima y del victimario, etc., pero no perciben la maldad ni la bondad, ya que no son objetos en el mundo que podamos percibir a través de nuestros sentidos.

Dice Hume, en sus Investigaciones sobre la moral:

La razón juzga los hechos o las relaciones. Investiguemos, pues, en primer término, dónde está el hecho que aquí llamamos delito; señalémoslo, determinemos el tiempo de su existencia, describamos su esencia o naturaleza, expliquemos el sentido o facultad ante la cual se descubre (2004, pp. 167-168) (cursivas en el original).

Así, los enunciados que contienen categorías morales, no nos hablan de lo que son las cosas, no describen nada. Entonces iqué es lo que hacen los enunciados morales? ¿De qué hablan? Según Hume, los enunciados morales son la manifestación de aprobación o desaprobación de acciones que suceden en el mundo. De tal manera que, cuando nuestros testigos del crimen, emiten la expresión: "matar es malo", lo que realmente están haciendo no es dando una descripción de las características del asesinato, sino expresando el sentimiento que genera en ellos el hecho.

Ahora bien, los enunciados morales hablan de cómo debe ser el mundo —al menos para el sujeto que los enuncia-, mientras que los descriptivos hablan de cómo es el mundo. Esta distinción, aunque obvia, es importante ya que, para Hume la naturaleza de ambos es tan disímil que no existe una relación directa ni necesaria entre ellos. La facultad humana que permite conocer el mundo no es útil para determinar lo correcto o lo incorrecto, lo justo o lo injusto. Por tanto, nuestro conocimiento de las cosas no determina de ninguna manera en nuestras opiniones morales. En el tercer libro del Tratado de la naturaleza humana, dice Hume:

[...] La moralidad no consiste en relaciones -objeto de la ciencia-, sino que, si se examina con cuidado [...] tampoco consiste la moralidad en ninguna cuestión de hecho que pueda ser descubierta por el entendimiento (1981, p. 668).

Pero aún hay más. Según Hume solamente pueden ser verdaderos o falsos los enunciados que hablen de los hechos del mundo o de las relaciones de ideas. Por tanto, y dado que los enunciados morales no son el resultado de ninguna de estas dos operaciones, no pueden ser ni verdaderos ni falsos. No existe ningún criterio objetivo, ni metodológico que nos permita saber la verdad o la falsedad de lo que consideramos correcto o incorrecto.

Por su parte, si bien el esfuerzo hecho por el positivismo lógico en sus estudios sobre ética iban dirigidos particularmente a esclarecer la naturaleza de los enunciados morales, lo que lo enmarcaría dentro de un análisis más de tipo metaético que propiamente ético, sus conclusiones son ampliamente relevantes para posteriormente dilucidar la naturaleza misma de la moral. 
Quizá el aporte más importante en la formulación del emotivismo positivista fue el realizado por Ayer en su libro Lenguaje, verdad y lógica. Allí, el filósofo británico expone las principales tesis positivistas, y la teoría del criterio de verificación. Dicho criterio será de capital importancia para el desarrollo general de las posiciones positivistas en general, y para el análisis del lenguaje moral del propio Ayer en especial. Más o menos, el slogan del criterio verificacionista es el siguiente: un enunciado es literalmente significativo si es a priori, o fáctico; es decir, un enunciado es verdadero o falso, en el caso de los enunciados a priori (enunciados de las matemáticas o de la lógica), gracias a la definición de los términos: si es tautológico es verdadero, si es contradictorio es falso. En el caso de los enunciados fácticos, la verdad o falsedad debe ser verificada por medio de la observación empírica (si el enunciado se corresponde con los hechos del mundo, entonces es verdadero; si no lo hace, entonces es falso). Sin embargo, hay otra serie de enunciados cuya verdad o falsedad no puede ser establecida, ya que no son ni a priori, ni fácticos, es decir, no son matemáticos ni lógicos, ni describen nada del mundo que pueda ser verificado a través de la observación, por tanto, que carecen de sentido. Entre estos últimos enunciados tenemos los enunciados éticos.

De tal manera que, nuevamente, cuando los testigos dicen: "matar es malo", su enunciado carece de criterios de verdad o falsedad dado que, no describe nada del mundo; carece de criterio de verificación. Ahora bien, cuando uno de los testigos dice que "el asesinato se cometió con un revolver" bastará con verificar si dicha expresión se corresponde con la realidad, para poder establecer si es verdadero o falso.

En concordancia con Hume, Ayer sugiere que las expresiones morales dan cuenta de las emociones que experimenta el sujeto ante cierto tipo de acciones. Por supuesto, estas expresiones no añaden nada al contenido fáctico de la acción, o del hecho del que se habla, simplemente deja en claro cuál es la valoración, o el grado de aprobación o desaprobación de quien la emite. Es decir que, si uno de los testigos dijera algo así como: "me parece muy malo que A haya asesinado a B", no está diciendo otra cosa que: "A asesinó a B".

Por su parte, Charles Louise Stevenson, en su libro de 1944 Ética y lenguaje (1984), hace algunos aportes sustanciales a la teoría emotivista. Según Stevenson la diferencia entre enunciados significativos y oraciones prescriptivas esconden, además, una diferencia entre tipos de desacuerdos a los que se pueden enfrentar dos sujetos que emiten aseveraciones contradictorias.

Volvamos al ejemplo. Los testigos no están de acuerdo con toda la información. Pueden estar en desacuerdo respecto al tipo de arma que se usó, en si el asesino tenía el arma en la mano derecha o izquierda, etc. Estos desacuerdos son desacuerdos respecto a las creencias que tienen cada uno acerca de hechos en el mundo y con base en dichas creencias emiten enunciados que son literalmente significativos, 
dado que su verdad o falsedad se puede establecer (así sea de manera hipotética) a través de una corroboración empírica. A este tipo de desacuerdos Stevenson los llama desacuerdos en las creencias.

Lo interesante de este tipo de desacuerdos es que cargan consigo la posibilidad de convertirse en acuerdos luego de que una corroboración empírica arroje un resultado acerca de la verdad o falsedad de los enunciados que suscitan el desacuerdo. Quienes aseguran que no hay vida en Marte se enfrentan a un desacuerdo en sus creencias respecto a quienes aseguran que sí la hay. Ese desacuerdo será dirimido en el momento en que podamos corroborar empíricamente si en efecto hay vida o no en dicho planeta.

Pero supongamos que uno de los testigos considera que el asesinato no es incorrecto gracias a que posee información que el otro testigo no posee. Así, ambos testigos pueden emitir juicios diferentes respecto al asesinato. La oración "considero correcto que A haya asesinado a B" riñe con una oración del tipo "considero incorrecto que A haya asesinado a B". Dado que ninguna de las dos oraciones es literalmente significativas ya que no describe ningún hecho en el mundo, lo que muestran es que ambos testigos poseen una actitud diferente respecto al asesinato de B. A este tipo de desacuerdos Stevenson los llama desacuerdos en las actitudes. Y ya que no es posible establecer ningún criterio de verdad acerca de estas aseveraciones, no existe ninguna garantía de que ambos testigos puedan convertir dicho desacuerdo en un acuerdo.

Sin embargo, ciertas creencias pueden afectar nuestras actitudes o nuestras valoraciones sobre hechos en el mundo. Advirtamos alguna información adicional acerca del crimen. Podemos decir, por ejemplo, que el asesinato se cometió por venganza, que el asesino nunca había cometido un crimen, que el muerto era un violador consuetudinario y que el victimario era el padre de una de sus víctimas. Ahora propongamos diferentes alternativas:

1. Supongamos que solamente uno de los dos testigos es informado acerca de los antecedentes del criminal y de la víctima. Puede suceder que el que posee la nueva información considere que, tratándose de una venganza perpetrada por el padre de una víctima de violación, el asesinato es bueno. En este caso, el desacuerdo en la creencia (los dos hombres tienen información diferente sobre el hecho), genera un claro desacuerdo en la actitud.

2. Pero podemos imaginar que, a pesar de tratarse de un violador reincidente, el testigo puede seguir considerando, al igual que su compañero que no conoce los antecedentes de la víctima, que el asesinato es malo. Aunque la información que poseen ambos difiera, la valoración de los hechos, y la actitud que asumen es la misma. 
3. Pero también podemos imaginar que ambos testigos poseen la misma información. Puede ser que, a la luz de la nueva evidencia, ambos estén de acuerdo en valorar positivamente el crimen; aunque igualmente puede suceder que, aun así, uno de ellos siga considerando que, sin importar las circunstancias, el asesinato es malo.

Pero hay algo más. La teoría emotivista de Stevenson sugiere que, detrás de toda expresión de aprobación o desaprobación de cualquier hecho, se esconde el interés por persuadir al interlocutor para que adopte una actitud similar. Es decir, cuando yo digo que "hacer X es malo", no solamente estoy asumiendo mi actitud acerca de hacer $X$, sino que, además, estoy intentando persuadir al otro de que, en efecto, "hacer $X$ es malo". De tal manera que decir, por ejemplo: "El asesinato es malo (desapruebo el asesinato, valoro negativamente el asesinato)" es lo mismo que decir: "Desapruebo el asesinato, deberías desaprobarlo tú también".

Como vemos, en todo juicio de valor está incluido el intento de convencer al otro de que la posición que asumimos es la mejor, y que debería adoptar la nuestra. Por supuesto, en una discusión que involucre un desacuerdo en las creencias, los interlocutores pueden apelar a esclarecer, o a tener una información más precisa acerca de lo que el mundo es, para convencerse de que adopten la posición propia. Sin embargo, como vimos hace un momento, no hay una relación causal necesaria que garantice que esto suceda.

Si bien buena parte de las objeciones a la teoría emotivista tienen estrecha relación con los presupuestos básicos del mismo positivismo lógico, de manera que se enmarcan con mayor precisión en el área de la filosofía del lenguaje, algunas otras objeciones fueron relevantes en el campo propiamente de la ética.

La primera que quiero mencionar es que la teoría ubica a las expresiones con contenido ético en el mismo saco de las expresiones de tipo metafísico, místico o teológico al ser todas ellas asignificativas. Sin embargo, ni siquiera los mismos positivistas intentaron negar la importancia y la vigencia de los enunciados valorativos. Podemos prescindir de expresiones como "el superhombre es un pasajero en trance del devenir" o "Dios es la unidad y la medida de todas las cosas"; pero no podemos prescindir de expresiones como "hacer X es moralmente bueno" o "lo correcto es hacer Y".

Por otra parte, la teoría emotivista queda vulnerable a cualquier objeción que se haga de la propia teoría verificacionista del significado; es decir, hay una absoluta dependencia entre ambas. De esta manera, el hecho de que las concepciones más ortodoxas y rígidas del positivismo lógico sean hoy cosa del pasado puede traer como inevitable consecuencia que el mismo emotivismo sea también cosa del pasado. 
Pero quizá la objeción más recurrente es que aceptar las tesis emotivistas implicaría claudicar en el propósito de establecer ciertos principios morales a través de un análisis racional. La validez o invalidez de una acción no puede ser determinada racionalmente ya que son solo el resultado de la emoción que genere y de la actitud que se asuma frente a ella. Esa idea contradice un tácito acuerdo en la historia de la filosofía moral (sobre todo a partir de la modernidad) según el cual la capacidad racional del ser humano puede permitirle establecer distinciones entre lo correcto y lo incorrecto. De hecho, teorías morales tan disímiles como el utilitarismo y la teoría kantiana comparten este mismo presupuesto. De manera racional, un sujeto puede establecer que tal acción es correcta o incorrecta en virtud del índice de beneficio general que pueda producir, en el caso del utilitarismo; o de si dicha acción se adecúa o no a una ley universal, a un imperativo categórico, en el caso kantiano. La teoría emotivista atenta contra la posibilidad romántica de establecer pautas de acción objetivas a las que se puede llegar gracias a la razón práctica. Es decir, la teoría emotivista nos puede dar una descripción de la naturaleza de la moral, pero no le es posible responder a la clásica pregunta de cómo debemos actuar, o que acciones son preferibles moralmente porque esa respuesta depende de cada sujeto gracias a las emociones que le despierte cada acción. Esta objeción conduce a una conclusión también interesante: aceptar el emotivismo es aceptar una forma de relativismo moral.

Además, la idea de que racionalmente podemos establecer aquellas acciones correctas y aquellas acciones incorrectas descansa sobre un presupuesto que vale la pena analizar: solo es posible hacer distinciones morales si se cuenta con un aparato racional que funcione con normalidad. El mismo Kant $(1999,2005)$ sugirió que si alguien era incapaz de establecer imperativos categóricos era porque su proceso racional no se había llevado a cabo de manera adecuada. Otra forma de ver el argumento es que la inobservancia moral es un problema racional.

De hecho, en los últimos 40 años, notables figuras de la filosofía han defendido distintas versiones de racionalismo moral en las que sostienen que la moral está basada en la razón práctica y que es posible dar razones acerca de si una acción es correcta o no lo es (T. Nagel en los años 70 y 80, E. Korsgaard en los 80 o P. Singer en los 90 y principios de este siglo).

Sin embargo, en el 2002, el filósofo norteamericano Shaun Nichols escribió el artículo Cómo los psicópatas amenazan el racionalismo ético o ¿es irracional ser amoral? que puede ser bastante esclarecedor al respecto.

Según Nichols hay dos tipos de concepciones racionalistas morales: un racionalismo que él llama racionalismo conceptual, y un racionalismo empírico. Por cuestiones de extensión, y por los intereses mismos de éste trabajo, solamente hablaré del segundo. 
La premisa sobre la que descansa el racionalismo empírico reza que "es un hecho empírico que los juicios morales humanos son una forma de juicios racionales, o lo que es lo mismo, nuestros juicios morales se derivan de nuestras facultades o capacidades racionales" (2002, p.9).

Recientemente, las teorías evolucionistas han aportado bastantes argumentos a favor del racionalismo empírico partiendo de una idea que parece tener mucho sentido: hay dos rasgos evolutivos que parecen distintivos del ser humano: 1) Nuestro aparato racional, o nuestras facultades racionales, y 2) lo que podemos llamar conciencia moral. Hasta donde sabemos, somos la única especie que posee estos rasgos, lo que conduce a pensar que puede haber una estrecha relación entre ambos (Tugendhat, 2001).

Peter Singer, por ejemplo, en su libro de 1995 How are we to live, desarrolló su propia versión de racionalismo empírico desde el punto de vista evolucionista, tratando de explicar en qué consiste el sentido de responsabilidad. Dice Singer:

¿Cómo puede la teoría evolucionista explicar el sentido de la responsabilidad para hacer de este mundo un lugar mejor? [...] He aquí una posible respuesta. Los seres humanos carecemos de la fuerza del gorila, del diente afilado del león, de la velocidad del guepardo. El poder del cerebro es nuestra especialidad. El cerebro es una herramienta para el razonamiento, y la capacidad de razonar nos ayuda a sobrevivir, a alimentarnos, a salvaguardar a nuestros niños (1995, p. 229).

Y más adelante continua Singer diciendo:

Nuestra capacidad natural para razonar nos permite distanciarnos de nuestro punto de vista y adoptar, en lugar de ello, una perspectiva más amplia; en última instancia, el punto de vista del universo mismo (229).

Así mismo como nuestra capacidad racional nos ha permitido un profundo entendimiento de las matemáticas, esa misma capacidad racional nos permite distinguir que cierto tipo de acciones son incorrectas y que cierto tipo de acciones son moralmente deseables.

De hecho, podríamos asumir que es una ventaja evolutiva el hecho de que los seres humanos hayamos desarrollado la capacidad de hacer distinciones morales gracias a otra ventaja evolutiva: la capacidad racional. Consideramos correcto cuidar a nuestros niños porque nuestra racionalidad nos permite entender que hay buenas razones para ello, particularmente nos permite entender que es un requisito para la preservación de nuestra especie. Por eso, cuando presenciamos actos atroces en contra de ellos nuestra racionalidad moral nos empujará a declarar estas acciones como incorrectas. 
Cuando uno de nuestros testigos del crimen emite en enunciado "el asesinato de B por parte de A es incorrecto" lo que está diciendo es que hay buenas razones para considerar dicho asesinato como incorrecto y esas razones las hallaremos (perdónenme la perogrullada) gracias a nuestra capacidad racional.

De esta manera, podríamos suponer que cualquier sujeto con un aparato racional perfectamente funcional podría eventualmente hacer las mismas distinciones morales que haría cualquier otro, así como cualquier persona racionalmente sana puede entender que $2+2$ es igual a 4 (este argumento no es distinto al argumento kantiano). Nichols se vale de los estudios en sicopatías para poner a prueba los principios del racionalismo empírico.

Si el argumento del racionalismo empírico es verdad entonces los psicópatas, al carecer de la capacidad de distinguir las acciones correctas de las incorrectas, carecen también de un aparato racional funcional, es decir, sus crímenes son el resultado de algún tipo de deficiencia o de fallo en su sistema racional.

Los estudios analizados por Nichols en sicología moral han arrojado como resultado que los seres humanos distinguimos, desde niños, entre violaciones morales y violaciones convencionales. Entre las violaciones morales podemos encontrar acciones como producirle dolor a alguien, no atender a nuestra responsabilidad respecto a las personas que están a nuestro cuidado, asesinar, etc., mientras que en las violaciones convencionales podemos encontrar acciones como pasarnos el semáforo en rojo, colarnos en una fila, etc.

Atendiendo al argumento del racionalismo empírico hay buenas razones para creer que cualquier sujeto racionalmente sano está en capacidad de entender que hay buenas razones para no hacerle daño a otro o no pasarse el semáforo en rojo y, con base en estos razonamientos, evitará lastimar a otros y detendrá su auto en el semáforo. También está en capacidad de entender que, si bien es preferible atender a las exigencias convencionales, es mucho más grave no atender a las exigencias morales.

Sin embargo, la evidencia, en el caso de los psicópatas (que son, quizá, el ejemplo más paradigmático de maldad que podemos encontrar) muestra algo diferente. Estudios como los de Turiel et al. (1987), Dunn \& Munn (1987), Smetana \& Braeges (1990), Nucci (1986), citados por Nichols (2002), han arrojado que cuando se le pregunta a asesinos seriales si sabe que hay razones para declarar que sus acciones son moralmente incorrectas, declaran que sí y el razonamiento que siguen para llegar a esta conclusión es el mismo razonamiento que haría una persona normal. La diferencia es que, a pesar de ello, al asesino no le importa. De hecho, estos estudios han mostrado que la mayoría de los sicópatas estudiados estaban en la capacidad de hacer operaciones matemáticas de un grado medio de complejidad (como la mayoría de la gente), y algunos de ellos podría incluso alcanzar altos grados de 
complejidad en sus conocimientos matemáticos, lo que parece ser una evidencia de que su sistema racional está intacto. Así mismo, el sicópata reconoce que es mejor detener el auto cuando el semáforo está en rojo, porque de lo contrario podría provocar un accidente, además de que haría la movilidad de una ciudad más caótica, sólo que no le importa hacerlo.

La diferencia entre los sicópatas y las personas comunes parece entonces estar en otro lado. Cuando se le cuestionó a los primeros si su asesinato les produjo algún sentimiento de aprehensión, su respuesta fue negativa. El hecho de que el asesinato no les produzca ninguna emoción hace que no se detengan cuando quieren hacerlo, a pesar de que racionalmente saben que hay razones para no hacerlo.

En su libro El error de Descartes (2008), publicado en 1996, el neurocientífico portugués Antonio Damasio expuso el caso de Phineas Gage, un responsable y solícito empleado minero. Gage sufrió un terrible accidente mientras intentaba poner una carga explosiva en una enorme roca. El explosivo detonó gracias a un descuido de su parte y una barra de hierro se incrustó en su cabeza entrando por su mejilla izquierda y saliendo por la zona frontal. Antes del accidente Gage tenía fama en su comunidad de ser un hombre respetuoso, atento a las normas y con una notable conciencia moral. Para sorpresa de todos, Gage sobrevivió al accidente y eventualmente se recuperó volviendo a su vida normal. Sin embargo, su vida ya no fue tan normal. A pesar de que sus capacidades racionales no disminuyeron en nada, el comportamiento de Gage cambió radicalmente. Como lo relata Damasio, dejó de atender a sus obligaciones laborales, se hizo desprolijo e irrespetuoso, usaba un lenguaje soez frente a las damas y cometió algunas acciones moralmente reprobables. Los estudios en neurociencia de Damasio, y el análisis del caso de Gage mostraron que la parte del cerebro que se vio afectada con el accidente no fue la parte con la que hacemos operaciones racionales, sino la zona donde se ubican las emociones. La lesión en el lóbulo prefrontal de Gage fue tan severa que a partir del accidente Phineas dejó de tener emociones. Lo curioso es que su ausencia de emociones coincide con una especie de indolencia o de inobservancia moral. Parecía que para Gage preferir las acciones correctas de las incorrectas no tenía mayor importancia.

Los casos de los sicópatas y de Gage parecen poner en duda el argumento del racionalismo empírico, pero, además, parecen indicar que lo que llamamos conciencia moral está más estrechamente ligada a nuestras emociones que a nuestra capacidad racional.

Pero también hay una razón que parece intuitiva: es natural que los seres humanos, cuando estamos en presencia de una acción que consideramos atroz o moralmente cuestionable generemos emociones sobre ellas. De hecho, observar un video en el que un miembro de Estado Islámico corta el cuello de una víctima 
indefensa produce en nosotros una emoción mucho antes de que podamos hacer racional las razones por las cuales esta acción es incorrecta.

Incluso, muchos de los protocolos investigativos en sicología moral y en neuroética consisten en exponer al sujeto experimental a imágenes que suponen una carga moral, como una persona golpeando indiscriminadamente a otra, o un intento de abuso sexual, y analizar cómo reaccionan ciertas zonas del cerebro a dichas imagen; particularmente, se analiza la actividad en las mismas zonas encargadas de las emociones, ya que son estas las que muestran actividad.

Podemos pensar, incluso que hemos desarrollado un mecanismo evolutivo que nos permite evitar ciertas acciones y preferir otras. Quizá, la razón por la cual tenemos sentimientos tan profundos por nuestros hijos es porque dichos sentimientos nos empujan a protegerlos, lo que jugaría a favor de la preservación de nuestra especie, o al menos de nuestro material genético.

De ser así, las bases del emotivismo humeano paracen responder mejor a las explicaciones en neurociencia de Damasio o a los estudios en sicología moral de los que se valió Nichols para tratar de mostrar que la moral no es racional.

Por supuesto, ni la teoría humeana ni la positivista se ajusta de manera plena a los hallazgos en neurociencia y en sicología evolutiva. Al margen de si lo bueno o lo malo son hechos en el mundo que puedan ser aprehendidos a través de alguna de las operaciones del entendimiento propuestas por Hume, o si los juicios morales son asignificativos, lo cierto es que la pretensión de ambas teorías de encasillar la naturaleza moral humana en el ámbito más de las emociones que de la razón, parecía estar bien encaminada.

Por supuesto, esto deja muchos interrogantes aún por resolver. Falta explicar entonces por qué la misma acción puede generar emociones distintas en sujetos distintos o si es de esperarse que las mismas acciones generen las mismas emociones en cualquier espectador. La emoción que siente el verdugo del Estado Islámico no es la misma que genera en nosotros. Quizá Stevenson tienen razón cuando argumenta que nuestras creencias pueden tener influencia en nuestras emociones o nuestras actitudes ante hechos morales. Pero lo importante aquí, creo yo, es que ubicar la naturaleza moral en el lugar que le corresponde puede conducir, eventualmente, a resolver estas y otras preguntas $\varphi$

\section{REFERENCIAS}

Ayer, A.J. (1985). Lenguaje, verdad y lógica. Barcelona: Orbis.

Damasio, A. (2008). El error de Descartes. Madrid: Booket. 
Damasio, A. (2010). En busca de Spinoza. Buenos Aires: Ediciones Destino.

Hume, D. (2012). Tratado sobre la naturaleza humana. Madrid: Tecnos.

Kant, I. (1999). Fundamentación de la metafísica de las costumbres. Madrid: Ariel.

Kant, I. (2005). Crítica de la razón práctica. México: FCE.

Korsgaard, C. (1986). Skepticism about Practical Reason. Journal of Philosophy, $83(1), 5-25$.

Mill, J.S. (2014). El utilitarismo. Madrid: Alianza.

Nagel, T. (1970). The Possibility of Altruism. Princeton, NJ: Princeton University Press.

Nichols, Sh. (2002). How Psychopaths Threaten Moral Rationalism, or Is it Irrational to Be Amoral? The monist, 2 (2), 285-303.

Piaget, J. (1966). The Psychology of Moral Development: The Nature and Validity of Moral Stages. New York: Free Press.

Rabossi, E. (1971). Emotivismo ético, positivismo lógico e irracionalismo. Diánoia, 17 (17); 36-61.

Singer, P. (1995). How Are We to Live? Amherst, NY: Prometheus Books.

Stevenson, Ch. L. (2004). "El significado emotivo de los términos éticos". El positivismo lógico. Bogotá: FCE.

Stevenson, Ch.L. (1984). Ética y lenguaje. Madrid: Paidos.

Tugendhat, E. (2001). Problemas. Madrid: Gedisa.

Von Wright, G.H. (2010). La diversidad de lo Bueno. Madrid: Marcial Pons.

Williams, B. (1981). "Internal and External Reasons," En Moral Luck. Cambridge: Cambridge University Press.

Zavadivker, N. (2008). Alfred Ayer y la teoría emotivista de los enunciados morales. Anuario Filosófico, XLI (3), 661-685. 